\title{
High-Dose Dexmedetomidine Promotes Apoptosis in Fetal Rat Hippocampal Neurons
}

\author{
Qiufeng Wei' \\ Jing Chen' \\ Fei Xiao (iD) ${ }^{\prime}$ \\ Youbing $\mathrm{Tu}^{2}$ \\ Yu Zhongl,* \\ Yubo Xie (D) ${ }^{1, *}$ \\ 'Department of Anesthesiology, The \\ First Affiliated Hospital of Guangxi \\ Medical University, Nanning, 53002I, \\ People's Republic of China; ${ }^{2}$ Department \\ of Anesthesiology, Shenzhen People's \\ Hospital, The First Affiliated Hospital of \\ Southern University of Science and \\ Technology, Shenzhen, Guangdong, \\ 518020, People's Republic of China \\ *These authors contributed equally to \\ this work
}

Correspondence: Yubo Xie; Yu Zhong Department of Anesthesiology, The First Affiliated Hospital of Guangxi Medical University, No. 6 Shuang-Yong Road, Nanning, 53002I, Guangxi, People's

Republic of China

Tel +86 18677958208

+86 I5I 77929679

Email xybdoctor@|63.com; zzlug|@|63. com
Objective: Dexmedetomidine (DEX) is a potent a2-adrenoceptor agonist that has sedative, analgesic, and anxiolytic effects. Its primary clinical use is as an adjunct to general anesthesia to reduce anesthetic doses, provide analgesia and sedation in the preoperative and postoperative periods, it also used in intensive care units (ICUs). However, high concentrations of DEX may have toxic effects on neurons and cause neuronal apoptosis. This study aimed to evaluate the potential proapoptotic effects of DEX on fetal rat hippocampal neurons.

Methods: Primary hippocampal were cultured in vitro for 8 days and incubated with different DEX concentrations for $3 \mathrm{~h}$. Cell viability was measured using cell counting kit- 8 assays. Cell apoptosis was evaluated using flow cytometry. The expression of apoptosisrelated proteins, such as cleaved caspase-3, caspase-9, Cyt-c, Bax, and Bcl-2, was measured by Western blotting. The mitochondrial ATP levels, $\Delta \psi \mathrm{m}$, and ROS analyzed were conducted.

Results: High concentrations of DEX $(\geq 100 \mu \mathrm{M})$ significantly reduced cell viability, induced neuronal apoptosis, upregulated the protein expression of cleaved caspase 3 , Bax, cleaved caspase 9, and Cyt-c. DEX also considerably promoted the release of ROS. However, DEX $(\geq 100 \mu \mathrm{M})$ downregulated the protein expression of Bcl-2, decreased the mitochondrial membrane potential (MTP), and reduced ATP synthesis.

Conclusion: High concentrations of dexmedetomidine produced toxic effects on neurons and caused neuronal apoptosis.

Keywords: dexmedetomidine, hippocampus, neurotoxicity, mitochondrial membrane potential, ROS

\section{Introduction}

With the development of anesthesia technology, millions of newborns and children receive general anesthesia during surgeries, imaging tests, and invasive procedures each year. However, whether general anesthetics have neuroprotective effects has been a controversial topic among scientists. Studies have shown that exposure to general anesthesia does not affect infant neurodevelopment. ${ }^{5-8}$ However, some studies have shown that general anesthesia exposure can increase the risk of long-term neurocognitive problems ${ }^{9-12}$ Preclinical animal studies, multi-center prospective studies, and large-scale retrospective studies have shown that general anesthetics may cause neurotoxicity in the developing brains of children, affecting the memory and learning abilities of these children. The Food and Drug Administration (FDA) of the United States issued a warning in December 2016 for the repeated or lengthy use of general anesthetics and sedation drugs in young children or women in their third trimester of 
pregnancy due to concerns regarding possible negative alterations in brain development. The study, published in JAMA, showed that higher doses of general anesthesia exposure and longer durations of anesthesia accumulation were associated with more toxic effects on neurons and lower white matter integrity in the corpus callosum associated with the observed impairment of neurocognitive function. Based on the neurotoxic effects of general anesthetics on specific populations such as children, it is urgent to study whether other sedatives can also produce neurotoxic effects. DEX is a highly selective type of 2-adrenergic receptor agonist with sedative, anxiolytic, and analgesic effects. The combination of DEX and general anesthesia can reduce the dose of the general anesthesia drugs used. Therefore, DEX is increasingly used for sedation in children based on these advantages, although there is no medication guidance for children. ${ }^{13-15}$ Previous studies have shown that DEX has neuroprotective effects on reducing cerebral ischemiareperfusion injury and can reverse neuronal apoptosis induced by general anesthesia exposure in newborn mice. ${ }^{6,11,16-18}$ However, the potential neurotoxicity of high DEX concentrations has been poorly studied. There are still many uncertainties about the widespread application of DEX and its safety, including whether the cumulative dose of DEX has a neurotoxic effect on the central nervous system when used for an extended period. Therefore, our study aimed to observe whether high concentrations of DEX exerted neurotoxic effects by pretreating primary fetal rat neurons with different concentrations.

\section{Materials and Methods}

\section{Hippocampal Neuron Culture}

Primary hippocampal neurons were isolated from fetal rat hippocampi from 16-18-day-old Sprague-Dawley embryos according to previously described experimental methods. The practical steps were as follows: First, pregnant rats were anesthetized with $1 \%$ isoflurane, and the uterus was rapidly exposed to remove the fetus under sterile conditions. After the fetus was anesthetized and decapitated, the hippocampus was exposed and separated from brain tissue under a stereomicroscope, shredded to approximately $1 \mathrm{~mm} \times 1 \mathrm{~mm} \times 1 \mathrm{~mm}$ in size, placed in a centrifuge tube, treated with an equal volume of $0.25 \%$ trypsin-EDTA solution (Beijing Solar Science \& Technology Co., Ltd., Beijing, China, Cat\# T1300) and digested for 15 minutes at $37^{\circ} \mathrm{C}$. The tissue was dissociated by repeated trituration through a fire-polished glass pipette to obtain a single-cell suspension. Then the singlecell suspension was seeded onto polylysine-coated plates (Sigma-Aldrich Chemical Co., St. Louis, MO, USA, Cat\# P4832) at a density of $1-2 \times 10^{6}$ cells $/ \mathrm{mL}$ in a plating medium containing 88\% DMEM/F12 (Thermo Fisher Scientific Inc., Waltham, MA, USA, Cat\# 11,320,082), 10\% FBS $\left(\right.$ Gibco $^{\circledR}$; Thermo Fisher Scientific Inc., Cat\# 10,099,141), 1\% glutamine (Sigma-Aldrich Chemical Co., Cat\# G7513) and 1\% penicillin/streptomycin (Beijing Solar Science \& Technology Co., Cat\# P1400) at $37^{\circ} \mathrm{C}$ in a humidified atmosphere of 5\% CO2 and 95\% room air. Four hours after inoculation, the medium was replaced with a serum-free maintenance medium that was not conducive to glial cells' growth or survival. The maintenance medium consisted of $96 \%$ neurobasal medium supplemented with $2 \%$ B27 $\left(50 \times\right.$, Gibco $^{\circledR}$; Thermo Fisher Scientific Inc., Cat\# 21,103,049), 1\% 200 mM L-glutamine (100×, Sigma-Aldrich Chemical Co., Cat\# G7513) and 1\% penicillin/streptomycin (100×, Beijing Solar Science \& Technology Co., Cat\# P1400), and half of the maintenance medium was replaced every 3 days. Cell growth was observed and recorded, including morphology, density, and protrusions, under an inverted microscope every day during culture. All experiments were performed at 8 days in vitro (DIV). Sprague-Dawley was obtained from the Animal Use and Care Committee of Guangxi Medical University. The experimental procedure and protocols were approved by the Animal Use and Care Committee of Guangxi Medical University (No. SCXK GUI 2003-0005) and were performed by the Guideline for Ethical Review of Animal Welfare (GB/T 35,892-2018).

\section{Hippocampal Neuron Identification}

MAP2 is preferentially located in nerve cells. In neurons, MAP2 exists in neuronal cell bodies and dendrites, but little or no immunostaining is detected in axons. Therefore, MAP2-positive cells can be considered as neurons. ${ }^{19}$ Hippocampal neurons (8 DIV) were identified with rabbit monoclonal antibodies against microtubule-associated protein 2 (MAP2) (Abcam, Cambridge, England, UK, Cat\# ab79757). Then were fixed with $4 \%$ paraformaldehyde (Beijing Solar Science \& Technology Co.) for 30 minutes at room temperature. The cells were washed with PBST and permeabilized with $0.1 \%$ Triton X-100 (Beijing Solar Science \& Technology Co.) after the paraformaldehyde was removed. Then, the cells were blocked with 5\% goat serum (Beijing Solar Science \& Technology Co.) for 30 minutes and were incubated with primary antibodies against 
MAP2 (dilution: 1:200) overnight at $4^{\circ} \mathrm{C}$, followed by incubation with horseradish peroxidase-labeled secondary antibodies for 10 minutes at room temperature. The immune reaction was visualized by incubating the cells with the chromogen 3,3'-diaminobenzidine. Cells were counterstained with Gill-2 hematoxylin and then imaged using a Leica (Wetzlar, Hessen, Germany) microscope.

\section{Experimental Groups and Processing}

The 8 DIV primary hippocampal neurons were randomly divided into 7 groups using a computer-generated chart as follows: Group C (control group), Group D0.1 (0.1 $\mu \mathrm{M}$ DEX group), Group D1 (1 $\mu \mathrm{M}$ DEX group), Group D10 (10 $\mu \mathrm{M}$ DEX group), Group D100 (100 $\mu \mathrm{M}$ DEX group), Group D200 (200 $\mu \mathrm{M}$ DEX group), and Group D300 (300 $\mu \mathrm{M}$ DEX group) (Figure 1). In Group C, the culture medium was replaced with a fresh maintenance medium. The cells in Groups D0.1, D1, D10, D100, D200, and D300 were treated with $0.1,1,10,100,200$, and $300 \mu \mathrm{M} \operatorname{DEX}(3 \mathrm{~h})$, respectively. Neuronal cell viability was measured by the cell counting kit-8 (CCK-8) assay, and neuronal cell death was analyzed by flow cytometry (Annexin V/propidium iodide). Western blotting was used to measure the expression of apoptosis-related proteins, such as cleaved caspase 3, Caspase-9, Cyt-c, Bax, and Bcl-2, in hippocampal neurons. We also assessed ATP levels, mitochondrial membrane potential, and reactive oxygen species (ROS). Samples in each group were analyzed at least in triplicate, and each group had at least three independent batch samples.

\section{Neuronal Cell Viability Evaluation}

Neuronal cell viability was determined by CCK-8 assays (Dojindo Molecular Technologies, Inc., Kumamoto, Japan, Cat\# CK04). Two hundred microliters of suspended cells were plated in a 96-well plate at a density of $1 \times 10^{6}$ cells/ well and preincubated for 8 days in a humidified incubator at $37^{\circ} \mathrm{C}$ with a $5 \% \mathrm{CO} 2$ atmosphere. Each group was incubated according to the method described above. After the experimental treatment, the culture medium was discarded and replaced with $200 \mu \mathrm{L}$ of fresh medium, and $10 \mu \mathrm{L}$ of the $\mathrm{CCK}-8$ reaction solution was added to each well of the plate. The absorbance at a wavelength of $450 \mathrm{~nm}$ was measured by a microplate reader (Thermo Fisher Scientific Inc.) after the culture plate was incubated with a $5 \% \mathrm{CO} 2$ atmosphere at $37^{\circ} \mathrm{C}$ for $4 \mathrm{~h}$.

\section{Apoptosis Evaluation}

Hippocampal neurons were inoculated into a 12-well culture plate coated with L-L-Polylysine at a density of $1 \times 10^{6}$ cells/well and were continuously cultured for 7 days. After the experimental treatment, the neuronal cells were harvested and digested with EDTA-free trypsin (Beijing Solar

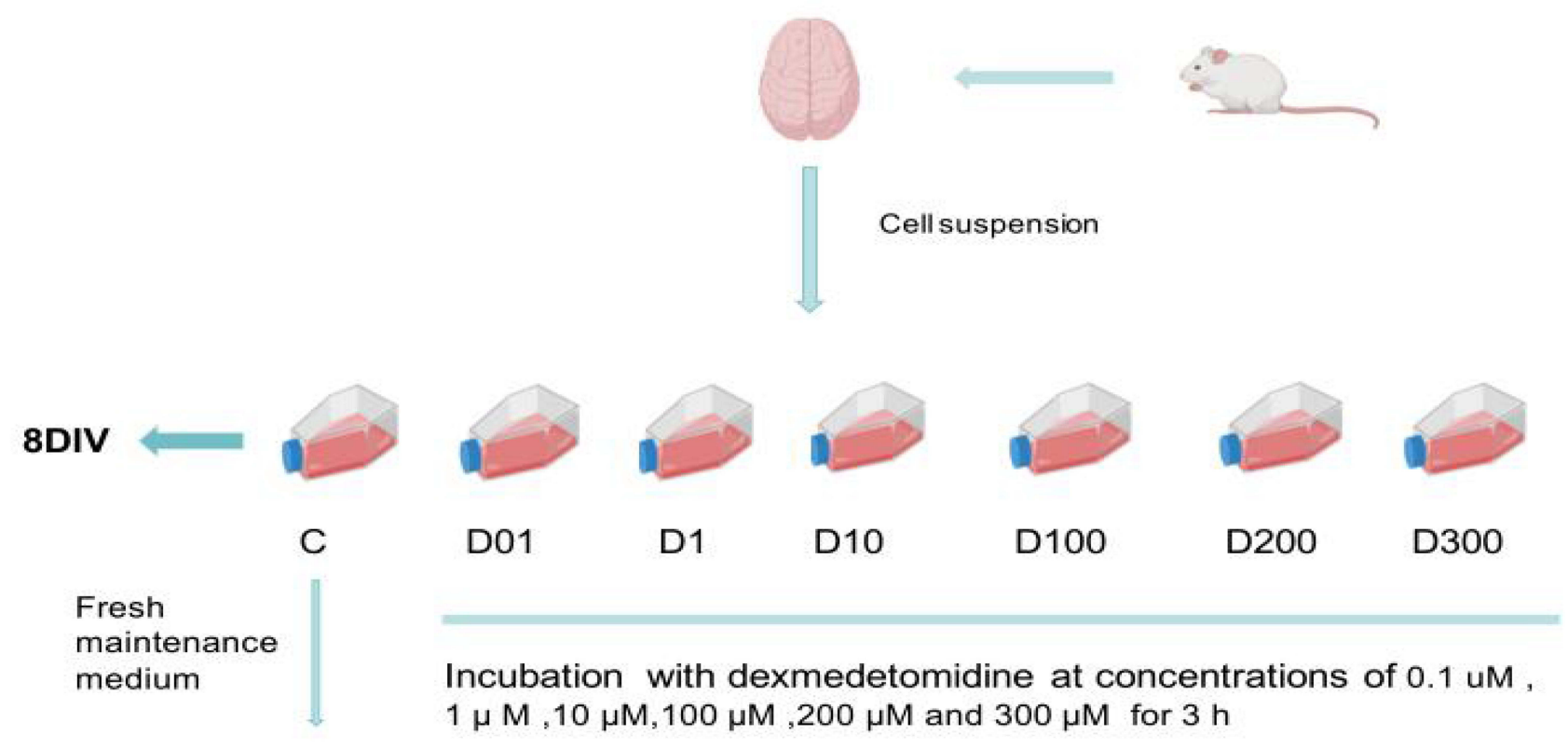

Figure I Experimental groups and processing.

Notes: The culture medium in group c was replaced with a fresh maintenance medium. The cells in Groups D0.I, DI, DI0, DI00, D200, and D300 were treated with 0.I $\mu \mathrm{M}, \mathrm{I} \mu \mathrm{M}, 10 \mu \mathrm{M}, 100 \mu \mathrm{M}, 200 \mu \mathrm{M}$ and $300 \mu \mathrm{M} \operatorname{DEX}(3 \mathrm{~h})$, respectively. 
Science \& Technology Co., Ltd., Beijing, China, Cat\# T1300), rinsed with PBS, and centrifuged at $1000 \mathrm{rpm}$ for 5 minutes at $4^{\circ} \mathrm{C}$. The supernatant was discarded, and $200 \mu \mathrm{L}$ of $1 \times$ binding buffer $(10 \times$ binding buffer diluted 10 times with PBS) was added. Then, the cells were stained with Annexin V/propidium iodide (BD Biosciences, Cat\# 556,547), and the apoptosis rate of cells in each group was analyzed by a FACSCalibur flow cytometer (BD Biosciences).

\section{Western Blotting}

Neuronal cells were scraped off the dish, then $120 \mu \mathrm{L}$ of lysis buffer (RIPA + PMSF + phosphatase inhibitor) was added and mixed with a pipette tip until the cells are completely lysed, then aspirated and placed in a frozen $1 \mathrm{~mL}$ EP tube and centrifuged at 12,000 rpm for 5 minutes at $4^{\circ} \mathrm{C}$. Each homogenate's protein concentration was determined using a BCA kit (Beijing Solar Science \& Technology Co.). The supernatant was collected and stored at $-80^{\circ} \mathrm{C}$. Loading buffer $(5 \times$, Beijing Solar Science \& Technology Co.) was added to each cell lysate sample, which was then boiled at $100^{\circ} \mathrm{C}$ for 5 minutes and separated on 12\% SDS-PAGE gels (Beijing Solar Science \& Technology Co.) in running buffer with 120 volts for approximately $1 \mathrm{~h}$, followed by transfer onto a polyvinylidene difluoride (PVDF) membrane ( $0.22 \mu \mathrm{m}$; EMD Millipore, Billerica, MA, USA). The membrane was blocked in 5\% skim milk and incubated at room temperature for $2 \mathrm{~h}$. Then, it was incubated at $4^{\circ} \mathrm{C}$ overnight with primary antibodies against cleaved caspase-3, cleaved caspase-9 (Cell Signaling Technology, Cat\# 9661), Cyt-c(Abcam, Cat\# ab32503), Bcl-2 (Abcam, Cat\# ab196495), Bax (Abcam, Cat\# ab32503), or GAPDH (Proteintech, Chicago, IL, USA, Cat\# 10,494-1-AP). The membranes were rinsed with Tris-buffered saline containing tween (TBST) (Beijing Solar Science \& Technology Co.) and then incubated with fluorescent dye-conjugated secondary antibodies (LI-COR Biosciences, Lincoln, NE, USA) for 3 h. Images were acquired by an Odyssey system (LI-COR Biosciences) and were quantitated with ImageJ.

\section{ATP Determination}

The culture medium was adsorbed, and 200 microliters of lysate were added to each well of the 6-well plate. Then, the medium was centrifuged at $4^{\circ} \mathrm{C}$ at $12,000 \mathrm{~g}$ for 5 minutes. The supernatant was removed, and the ATP detection reagent was diluted with the dilution solution at a ratio of 1:9. Next, $100 \mu \mathrm{L}$ of ATP detection solution was added to the detection well and incubated at room temperature for $3-5$ minutes. Then, $20 \mu \mathrm{L}$ of the sample was added to the test well and mixed quickly with a pipette. After 2 seconds, the RLU value or CPM was determined with a luminometer or a liquid flash instrument.

\section{Mitochondrial Membrane Potential}

The culture medium was removed, and $1 \mathrm{~mL}$ of JC-1 staining solution was added to the well. Then, the cells were incubated at $37^{\circ} \mathrm{C}$ for 20 minutes. During the incubation, an appropriate amount of JC-1 staining buffer $(1 \times)$ was prepared at a ratio of $4 \mathrm{~mL}$ of distilled water to $1 \mathrm{~mL}$ of JC-1 staining buffer $(5 \times)$ and placed in an ice bath. After incubation at $37^{\circ} \mathrm{C}$, the supernatant was removed, and the cells were washed twice with JC- 1 staining buffer $(1 \times)$. A total of $2 \mathrm{~mL}$ of cell culture medium was added, and the cells were observed under a fluorescence microscope.

\section{Intracellular ROS Analysis}

The DCFH-DA probe and fluorescence microscopy were used to analyze intracellular ROS levels. DCFH-DA was diluted with the serum-free medium at a ratio of 1:1000 to a final concentration of $10 \mu \mathrm{M}$. The cell culture medium was removed, and an appropriate volume of diluted DCFH-DA was added. The cells were incubated in a $37^{\circ}$ $\mathrm{C}$ incubator for 20 minutes. The cells were washed three times with a serum-free cell culture medium to sufficiently remove the DCFH-DA that did not enter the cells. The fluorescence intensity was observed under a laser confocal microscope (488 $\mathrm{nm}$ excitation wavelength, $525 \mathrm{~nm}$ emission wavelength) and analyzed by software.

\section{Statistical Analysis}

SPSS 22.0 (IBM Corporation, Armonk, NY, USA) and GraphPad Prism 6.07 software (OriginLab, Northampton, MA, USA) were used for statistical analyses. The Student's $t$-test was used to compare two experimental groups, and one-way ANOVA was used to compare several treatment groups. When appropriate, Bonferroni's post hoc comparison test was used. P-values, 0.05 were considered statistically significant.

\section{Results}

The Cumulative Dose of DEX Affects Hippocampal Neuronal Viability and Promotes Apoptosis

To determine the role of DEX in hippocampal neuronal viability, we examined the effects of DEX by CCK- 8 assays and flow cytometry. The results indicated no 
significant differences between group D01, group D1, and group D10 compared with the control group. A significant decrease in hippocampal neuronal activity was observed after hippocampal neurons were exposed to high DEX concentrations $(\geq 100 \mu \mathrm{M})$ (Figure $2 \mathrm{~A}$ and $\mathrm{B})$. The CCK-8 assays revealed that treatment with $100 \mu \mathrm{M}$ DEX markedly reduced neuronal viability by $47.44 \%$ compared with Group C. We also found the percentage of apoptotic cells was significantly increased in group D100, groupD200, and groupD300 (Figure 3).

\section{High-Dose DEX Enhanced Neuronal Apoptosis-Related Protein Expression}

In the present study, we found no significant differences between group D01, group D1, and group D10 compared with group C (Figure 3A-E). Group D100 and group D200, which were treated with high doses of DEX (100 $\mu \mathrm{M}$ and $200 \mu \mathrm{M}$ ), exhibited significantly increased protein expression of cleaved caspase 3, Caspase-9, Cyt-c, and Bax but decreased protein expression of Bcl-2 compared with that of the control group (Figure $3 \mathrm{~A}-\mathrm{E}$ ). In addition, we also observed that the protein expression of cleaved caspase 3, Caspase-9, Cyt-c, and Bax were significantly increased, and the protein expression of Bcl-2 was decreased in group D100 and group D200 compared with that of group D10 (Figure 3A-E). Moreover, $200 \mu \mathrm{M}$ DEX notably reduced the protein expression of $\mathrm{Bcl}-2$ (Figure 3A-E) and increased the protein levels of cleaved caspase 3, Caspase-9, and Cyt in hippocampal neurons compared with those of cells treated with a concentration of $100 \mu \mathrm{M}$. This finding suggests that increasing the cumulative dose of DEX enhanced neuronal apoptosis-related protein expression.

\section{High-Dose DEX Reduces Neuronal ATP and Increases ROS Levels}

The cumulative dose of DEX had a significant effect on cellular ATP and ROS levels. Our experimental results showed that $100 \mu \mathrm{M}$ and $200 \mu \mathrm{M}$ DEX significantly reduced neuronal ATP (Figure 4A) and increased ROS levels compared with group C (Figure 4B). Also, we observed that $100 \mu \mathrm{M}$ and $200 \mu \mathrm{M}$ DEX significantly reduced neuronal ATP and increased ROS levels compared with that of $10 \mu \mathrm{M}$ DEX (Figure 4A and B). DEX at 0.1, 1 , and $10 \mu \mathrm{M}$ exhibited no significant differences in neuronal cell ATP and ROS levels than group C. This finding shows that the increased cumulative doses of $100 \mu \mathrm{M}$ and
$200 \mu \mathrm{M}$ DEX resulted in significantly elevated ROS levels and decreased ATP levels.

\section{High-Dose DEX Reduces Cell Mitochondrial Membrane Potential}

No mitochondrial membrane potential damage or changes were observed in response to $0.1 \mu \mathrm{M}, 1 \mu \mathrm{M}$, and $10 \mu \mathrm{M}$ DEX (Figure 5). However, cumulative doses of $100 \mu \mathrm{M}$ DEX and $200 \mu \mathrm{M}$ DEX significantly reduced the cell mitochondrial membrane potential. Besides, doses of 200 $\mu \mathrm{M}$ DEX significantly reduced the cell mitochondrial membrane potential compared with $100 \mu \mathrm{M}$ DEX (Figure 5). This finding suggests that a cumulative dosedependent reduction in the cell mitochondrial membrane potential was observed.

\section{Discussion}

All commonly used general anesthetics, including sevoflurane, the most frequently used anesthetic in pediatric practice, have been found to cause widespread apoptotic neuronal death and to alter the dendritic architecture and deficits in long-term cognitive and behavioral function in animals, including small rodents and nonhuman primates, after exposures of up to six $\mathrm{h}$ early in life $\mathrm{e}^{20-26}$ Despite a lack of solid affirmative findings in human studies, additional research into better understanding developmental anesthetic neurotoxicity are urgently needed. Accordingly, the present study was designed to observe whether DEX promoted neuronal apoptosis in a dosedependent manner. Based on our current findings, this is the first study to demonstrate that treatment with highconcentration DEX $(\geq 100 \mu \mathrm{M})$ can promote neuronal apoptosis in vitro. Studies had shown that neuronal apoptosis in the hippocampus was observed when the concentration of DEX was greater than $5 \mu \mathrm{g} / \mathrm{kg}$. ${ }^{16}$ DEX produced significant cellular degeneration and apoptosis in primary sensory brain regions in the brains of 7-day-old rat pups. The higher DEX dose, $45 \mu \mathrm{g} / \mathrm{kg}$, resulted in numerically higher numbers of apoptotic and degenerated cells than the $30 \mu \mathrm{g} / \mathrm{kg}$ dose in almost all areas. These results showed that DEX promoted neuronal apoptosis in a dosedependent manner. ${ }^{27}$ Liu et al $^{17}$ found that cumulative doses of 50 to $250 \mu \mathrm{g} / \mathrm{kg}$ DEX in seven-day-old SpragueDawley rats could induce neuronal apoptosis in the somatosensory cortex. As shown in other in vivo studies, 30 $\mu \mathrm{g} /$ $\mathrm{kg}$ DEX can induce the Caspase 3-mediated immune response in neurons in the thalamic region (VPM, MG) 


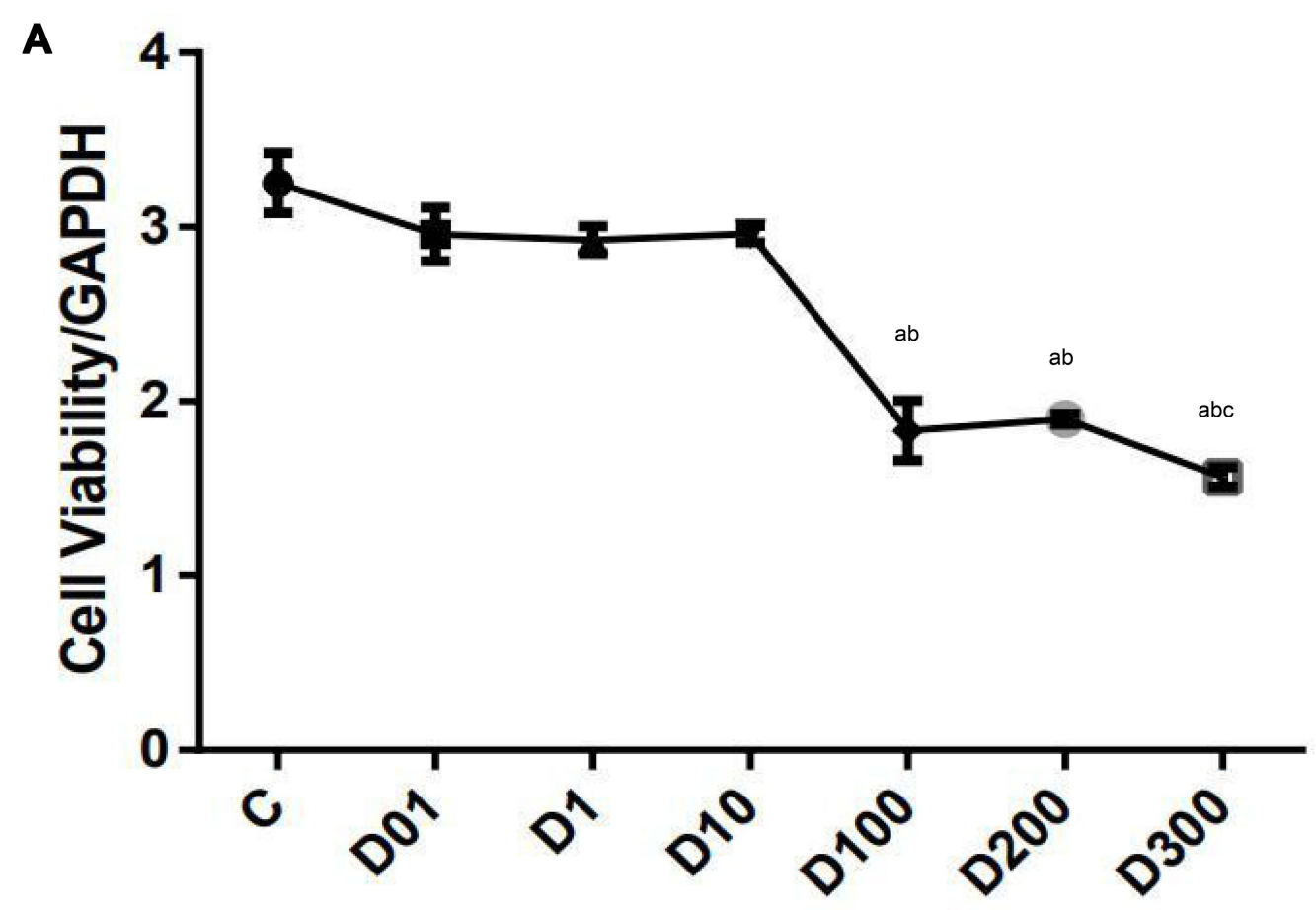

B

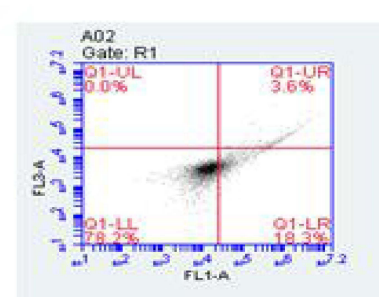

C

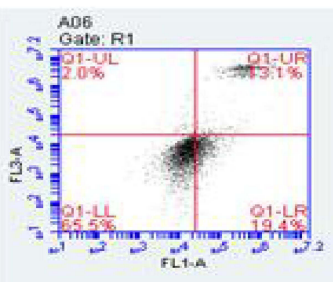

P01

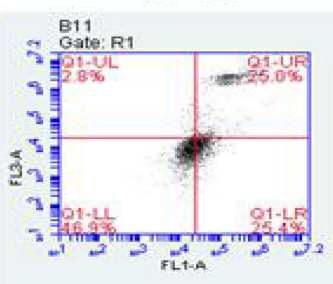

$\mathrm{P} 100^{\mathrm{ab}}$

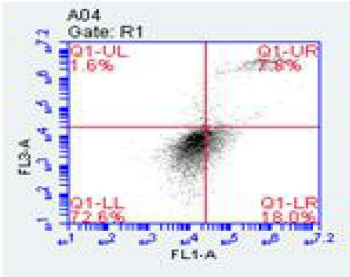

P0001

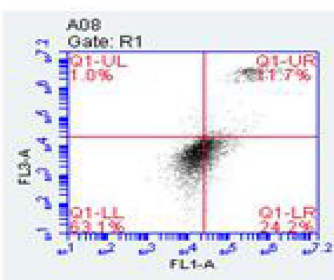

P1

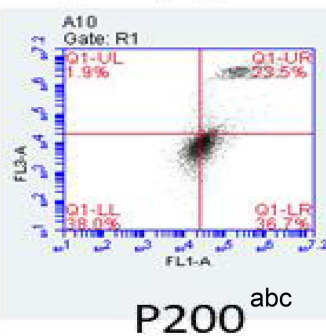

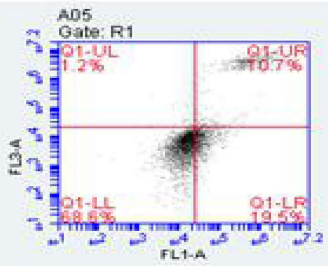

P001

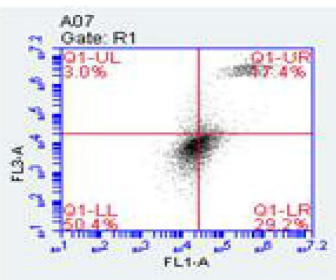

P10

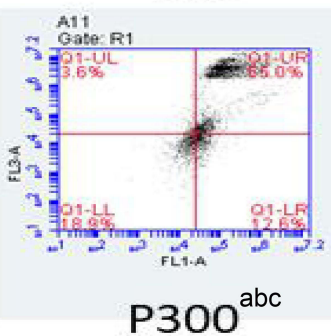

Figure 2 High concentrations of DEX $(\geq 100 \mu \mathrm{M})$ reduce neuronal viability and promote apoptosis.

Notes: (A) There were no significant differences between group D0I, DI, and DI0 compared with the control group (one-way ANOVA, ap $>0.05$, $n=18$ ), while a significant decrease in hippocampal neuronal activity was observed after hippocampal neurons had been exposed to high concentrations of DEX ( $\geq 100 \mu M$ ) (group DI00, D200 and D300; one-way ANOVA, ${ }^{b} P<0.05, n=18$ ). (B) There was no significant difference in hippocampal neuronal apoptosis between group $C$ and groups $D 0 I$, DI, and $D I 0$. However, significant increase in the percentage of apoptotic cells in group DI00, D200, and D300 was observed (one-way ANOVA, ${ }^{a} P<0.05$, $n=9$ ). ${ }^{a} P$ : compared with group $C$, ${ }^{b} P<0.05$; $P$ : compared with group $D I 0, P<0.05$; ${ }^{C}$ : compared with group $D I 00, P<0.05$. 
A

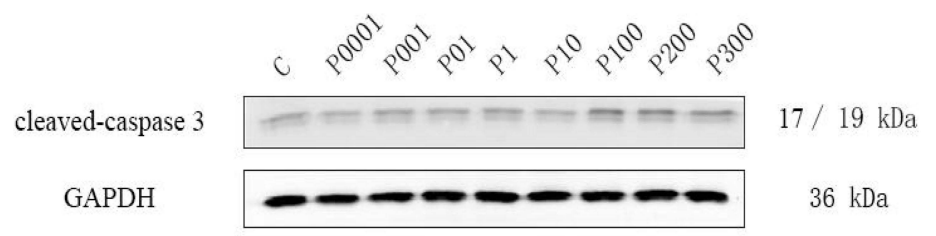

Cleaved Caspase-9

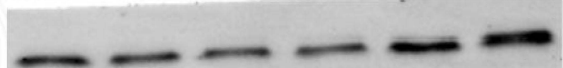

$38 \mathrm{KD}$

Bcl-2

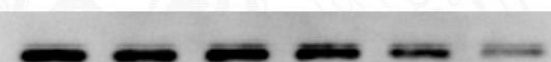

$26 \mathrm{KD}$

Bax

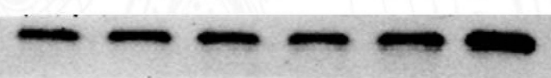

$20 \mathrm{KD}$

Cytc

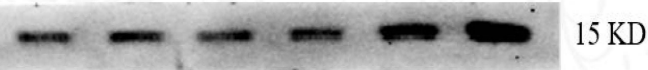

GAPDH

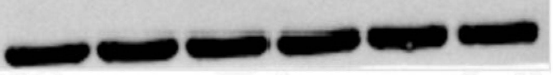

$36 \mathrm{KD}$

C D0.1 D1 D10 D100 D200

B

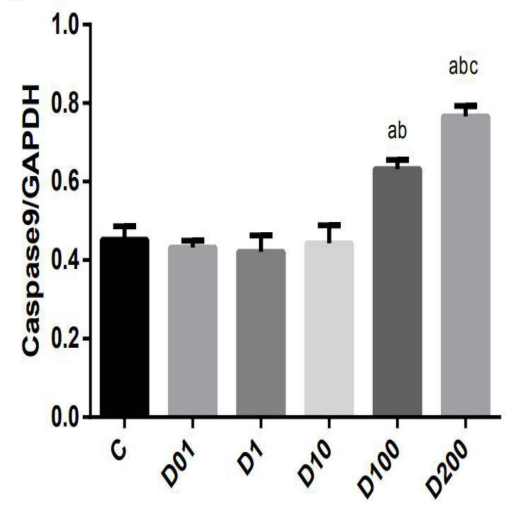

C
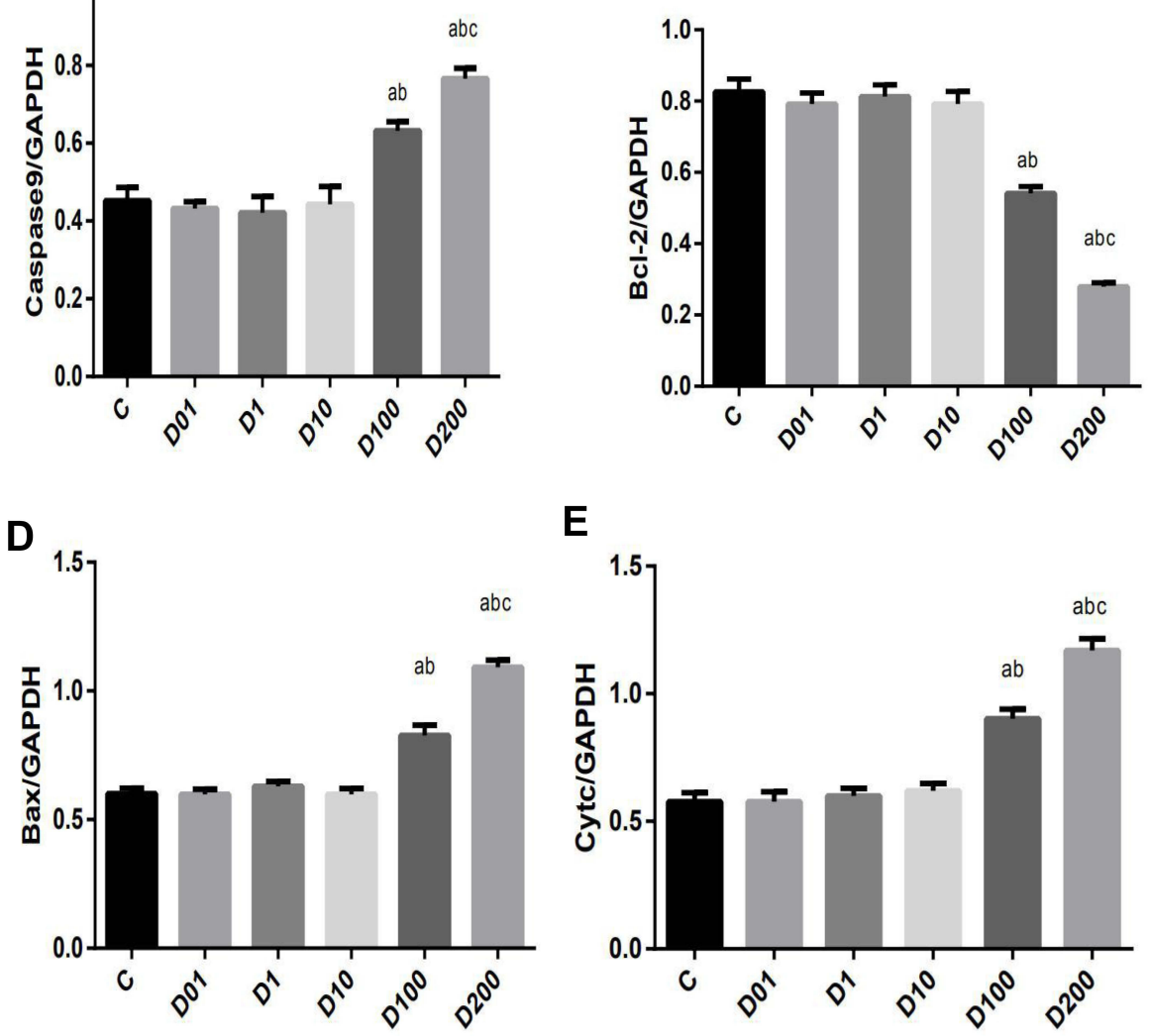

E

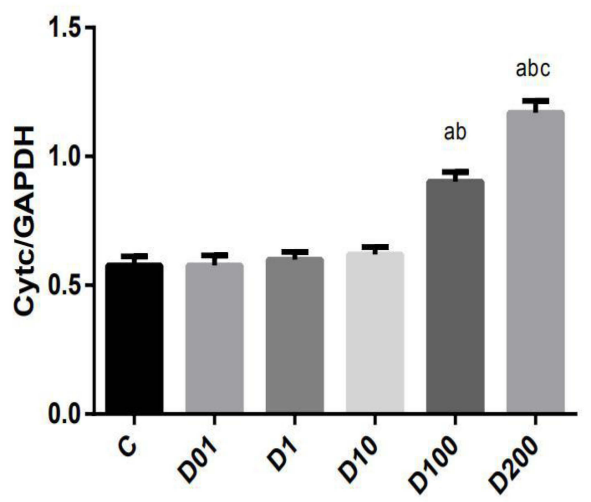

Figure 3 DEX promotes neuronal apoptosis-related protein expression in a dose-dependent manner.

Notes: (A-E) DEX $(100 \mu \mathrm{M})$ notably reduced the protein expression of the Bcl-2 and increased the protein expression of cleaved caspase 3, Caspase-9 and Cyt-c compared with that of group $C$ (one-way ANOVA, ${ }^{a} P<0.05, n=5$ ) and group DIO (DEX 10 $\mu M$ ) (one-way ANOVA, ${ }^{b} p<0.05$, $n=5$ ). There were no significant differences between group D0I (DEX 0.I $\mu \mathrm{M})$, DI (DEX I $\mu \mathrm{M})$ and DI0 (DEX I0 $\mu \mathrm{M})$ compared with group C(one-way ANOVA, $\left.{ }^{a} P>0.05, \mathrm{n}=5\right)$. DEX $(200 \mu M)$ notably reduced the protein expression of $\mathrm{Bcl}-2$ and increased the protein expression of cleaved caspase 3, Caspase- 9 and Cyt-c compared with that of the DEX I00 group (one-way ANOVA, ${ }^{c} P<0.05$, $n=5$ ). 

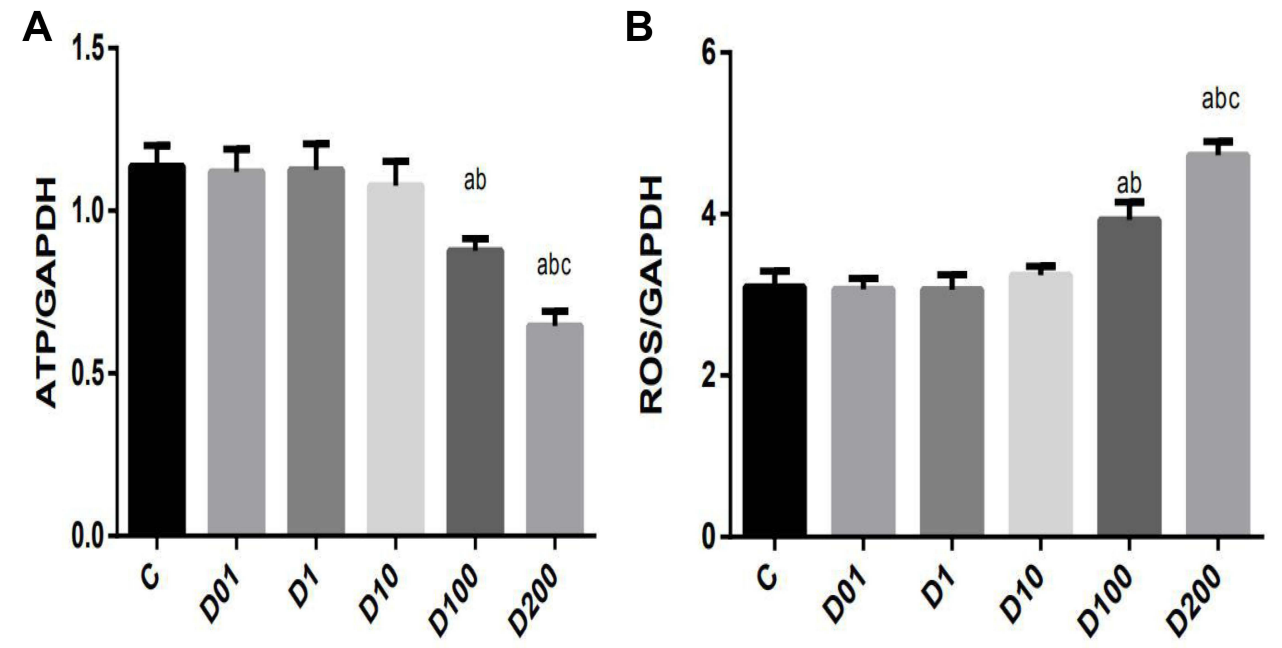

Figure 4 High concentrations of DEX reduce neuronal ATP and increase ROS levels.

Notes: (A and B) No significant differences were observed in neuron cell ATP and ROS levels between the D0.I, DI, and DI0 groups compared with group C (one-way ANOVA, ${ }^{a} P>0.05, n=9$ ). The dose of $100 \mu M$ DEX notably reduced neuronal ATP and increased ROS levels compared with those of group $C$ (one-way ANOVA, aP $<0.05$, $\mathrm{n}=9$ ). Significant differences were observed in neuronal ATP and ROS levels at doses of $10 \mu \mathrm{M}$ and $100 \mu M$ DEX (one-way ANOVA, ${ }^{b} P<0.05$, $n=9$ ). Furthermore, $200 \mu M$ DEX significantly elevated ROS levels and decreased ATP levels compared with $100 \mu M$ DEX (one-way ANOVA, ${ }^{c} P<0.05, n=9$ ). ${ }^{a} P$ : compared with group $C, P<0.05$; ${ }^{b} P$ : compared with group $D I 0, P<0.05$; ${ }^{c} P$ : compared with group $D I 00, P<0.05$.

and sensory cortex (S1, A1, V1) of newborn mice, and a large number of silver-stained cells can also be observed in these regions. Lee et $\mathrm{al}^{16}$ found that significant apoptosis could be kept in the thalamic posterior ventral nucleus and hippocampal CA2/3 neurons when the dose of DEX was $300 \mu \mathrm{g} / \mathrm{kg}$. Our previous study showed that $10 \mu \mathrm{M}$ DEX could effectively inhibit propofol's neurotoxic effect on primary hippocampal neurons without producing additional harmful effects, which was consistent with the observation in this study that no neuronal apoptosis was observed after incubation with $10 \mu \mathrm{M} \mathrm{DEX.}{ }^{28}$ In the present study, we observe that DEX at low concentrations had no damaging effect on neurons. In contrast, a high concentration of DEX $(\geq 100 \mu \mathrm{M})$ promoted neuronal apoptosis in a dose-dependent manner, Which further confirmed the neurotoxicity of DEX at high concentrations as previously reported.

Mitochondria are essential organelles in cells, whose primary function is to convert nutrients into ATP, which is used to maintain cellular metabolism ${ }^{27}$ large number of studies have confirmed that mitochondria play an essential role in regulating the process of apoptosis, which is closely related to the occurrence and development of many crucial apoptotic events ${ }^{29}$ Cytochrome $\mathrm{C}$ is not only a critical substance associated with electron transfer in the respiratory chain but also a significant regulator of apoptosis. The Cyt c-cysteine protease cascade is one of the main pathways that mediate apoptosis, promoting caspase-3 activation and apoptosis through its family members. When the mitochondrial structure is destroyed, Cyt-c can be released from the mitochondria into the cytoplasm ${ }^{30,31}$ to form a complex with apoptotic protease activator -1 (apaf-1) and apoptotic bodies. The poly-Cyt-c-Apaf-1 complex activates procaspase-9. Caspase- 9 induces the caspase- 6 and caspase- 3 cascades and ultimately promotes apoptosis $^{28,32} \mathrm{Bcl}-2$ group proteins mainly include the proapoptotic proteins $\mathrm{BAK}$ and $\mathrm{BAX}$ antiapoptotic protein BCL-2, and these proteins are primarily distributed on the mitochondrial membrane. ${ }^{33,34}$ Bax promotes Bax the opening of the mitochondrial permeability transition pore (PTP). The opening of the PTP can form a positive feedback amplification reaction, leading to disturbances in mitochondrial structure and function, external mitochondrial flow, potential membrane collapse, osmotic imbalance, and mitochondrial swelling and lysis, promoting the release of cytochrome $\mathrm{C}$ and the activation of caspase- 3 and eventually inducing apoptosis. ${ }^{35}$ In our study, primary hippocampal neurons in fetal mice were used as models of brain development. An evident decrease in neuronal cell viability and an increase in the apoptotic proteins Bax, cleaved caspase 9, cleaved caspase 3, and Cyt-c were observed in neurons that had been pretreated with 100 $\mu \mathrm{M}(>100 \mu \mathrm{M}) \mathrm{DEX}$. We inferred that a high concentration of DEX had a damaging effect on neuronal mitochondria, affecting the respiratory chain reaction, promoting the 


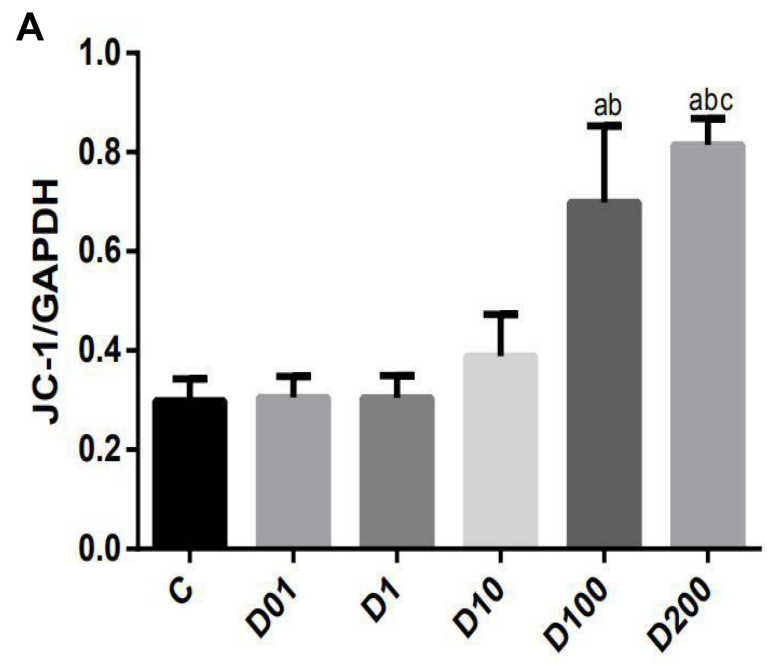

B

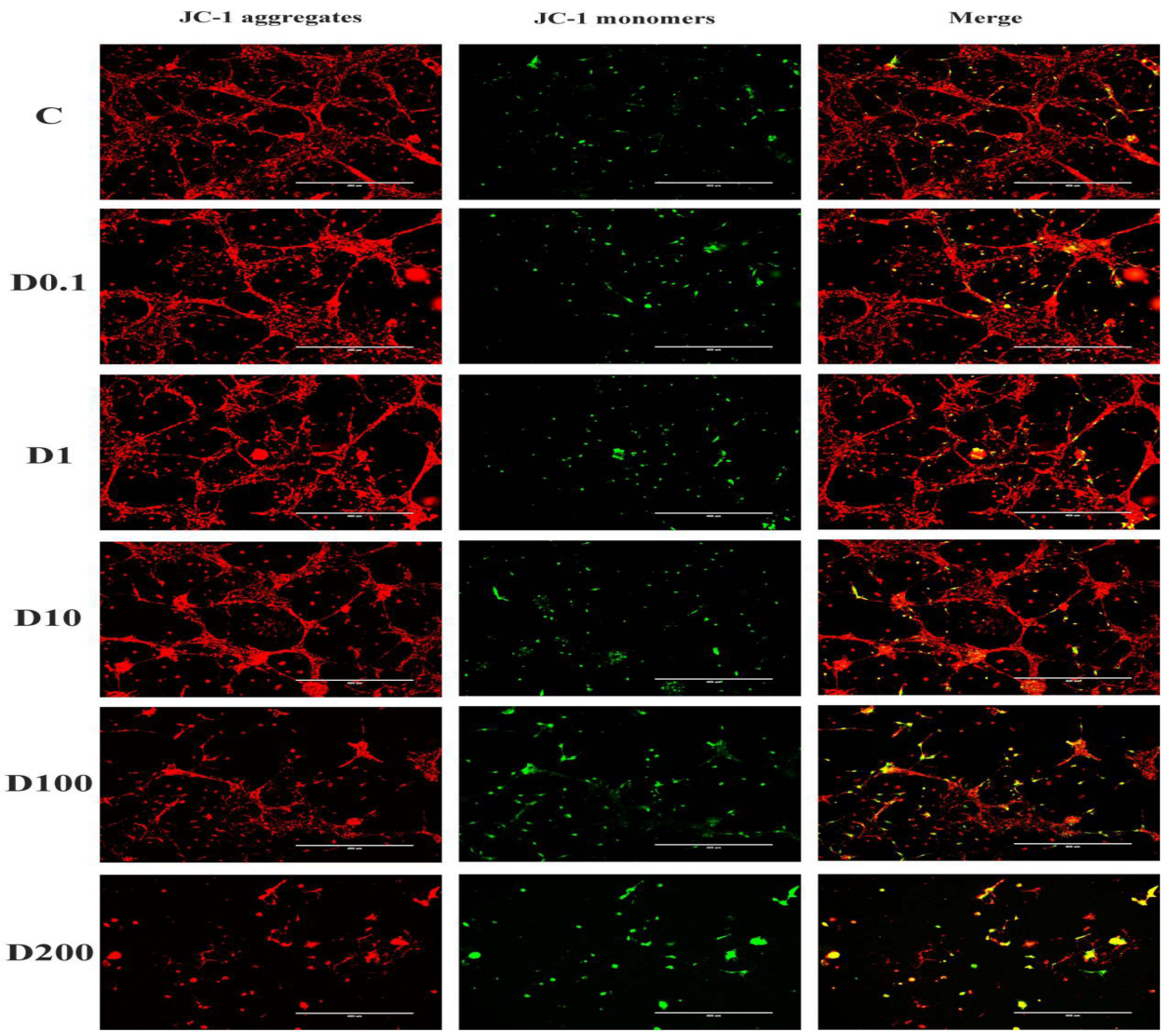

Figure 5 High-dose DEX reduces cell mitochondrial membrane potential.

Notes: (A) There was no mitochondrial membrane potential damage or changes in response to $0.1 \mu M, 1 \mu M$ and $10 \mu M$ DEX (one-way ANOA, ${ }^{a}>>0.05, n=9$ ). Increasing the concentration of DEX $(100 \mu \mathrm{M}, 200 \mu \mathrm{M})$ significantly reduced the cell mitochondrial membrane potential. DEX at $100 \mu \mathrm{M}$ and $200 \mu \mathrm{M}$ markedly decreased the cell mitochondrial membrane potential compared with that of group $C$ (one-way ANOVA, ${ }^{a} P<0.05, n=9$ ). DEX at $200 \mu M$ significantly reduced the cell mitochondrial membrane potential compared with that of cells treated with $100 \mu \mathrm{M}$ DEX (one-way ANOVA, ${ }^{c}>0.05, n=9$ ). (B) When the mitochondrial membrane potential is high, JC-I accumulates in the mitochondrial matrix to form polymers (J-aggregates) and produces red fluorescence. When the mitochondrial membrane potential is low, JC-I cannot be concentrated in the mitochondrial matrix. In this context, JC-I is a monomer and can produce green fluorescence. Treatment with $100 \mu \mathrm{M}$ and $200 \mu \mathrm{M}$ DEX reduced the level of red fluorescence and increased the level of green fluorescence compared with those of group C, DI and DI0, which indicates that the mitochondrial membrane potential is reduced. ${ }^{a}$ : compared with group C, $P<0.05$; ${ }^{b}$ : compared with group $D I 0, P<0.05$; ${ }^{C} P$ : compared with group $D I 00, P<0.05$. 
release of Cyt-c, and ultimately promoting neuronal apoptosis.

ATP is a kind of high-energy phosphoric acid compound. ROS are the products of oxygen metabolism in mitochondrial electron transmission, which helps to maintain cellular homeostasis and participate in cell signal transduction. However, too much ROS can oxidize DNA, proteins, and fatty acids. ${ }^{18}$ ROS is closely related to the activation of the mitochondrial-mediated apoptosis pathway. ${ }^{29}$ After Cyt-c is released from mitochondria, ROS production is increased, and apoptosis is more evident because the respiratory chain's electron transfer process is blocked. ${ }^{14}$ To verify whether DEX caused neuronal damage by acting on mitochondria, we also measured changes in ATP and ROS levels in neurons and changes in mitochondrial membrane potential in response to different DEX concentrations. The results showed that ATP levels significantly decreased and ROS levels increased dramatically in the D100 and D200 groups. Our results support Liu et al that a high concentration of DEX promotes neuronal cell necrosis and apoptosis. ${ }^{17}$ We also examined the effect of DEX on neuronal mitochondrial membrane potential and found that $>100 \mu \mathrm{M}$ DEX (including $100 \mu \mathrm{M}$ ) induced a significant decrease in mitochondrial membrane potential. Studies show that Bcl-2 family proteins can effectively prevent $\Delta \psi \mathrm{m}$ depolarization and inhibit apoptosis ${ }^{36-38}$ We also found that the $\mathrm{Bcl}-2$ protein was significantly reduced in group D100 and group D200, further confirming that the highconcentration DEX has a neurotoxic effect.

To date, The mechanisms by which high concentrations of DEX exert neurotoxicity remain unclear. In mammalian mitochondria, ROS mainly originates from NADH (ubiquinone oxidoreductase (complex I)) and ubiquinol (cytochrome c oxidoreductase (complex III)) of the electron transport chain. ${ }^{41}$ Complex I and complex III are the critical sources of ROS production. However, excessive ROS in geese can have toxic effects on cells. ${ }^{39,40}$ We found that the ATP levels were significantly decreased and that the ROS and Cyt-c levels were significantly increased in the D100 and D200 groups. We inferred that DEX exerts neurotoxic effects by inducing mitochondria-derived ROS production via the inhibition of inhibiting complex I and complex III of the mitochondrial respiratory chain, ultimately inducing cell apoptosis. In addition to activation of a2-adrenergic receptors, DEX-induced block of these currents could thus be potential mechanisms through which it may depress neuronal excitability. ${ }^{42}$ Shirasaka et al found that DEX increases G-protein-coupled inwardly, rectifying $\mathrm{K}+$ current and inhibits hyperpolarization-elicited inward current, ${ }^{43,44}$ Besides, Voltage-gated $\mathrm{K}+(\mathrm{KV})$ channels play an essential role in determining the excitability of neurons. Chen et al showed that DEX (30mM) significantly decreased current amplitude at the end of the voltage pulses from 2018 (152) to 1196 (81) pA, they found DEX produces a depressant action on IK(DR) in a concentration and statedependent fashion. We found that a high concentration of DEX significantly reduced neuronal excitability and thus inferred that it affected the action potential of neuron cells and reduced the excitability of nerve cells by inhibiting the opening of the $\mathrm{I}_{\mathrm{K}(\mathrm{DR})}$ channel, thereby directly regulating membrane ionic currents, modifying the mitochondrial membrane potential and apoptotic changes, and eventually triggering apoptosis.

Given the abundant confounders affecting young children requiring surgery with anesthesia, the ethical limitations of clinical studies, and the substantial differences between all model species and clinical practice. Although our present study showed that DEX promoted neuronal apoptosis in a concentration of $\geq 100 \mu \mathrm{M}$ in vitro, the DEX concentration used in this work could not be clinically achievable, it remains unresolved whether results from animal studies can be directly applied to pediatric anesthesia practice. It is prudent not only to identify safer, alternative anesthetic techniques in animals but also to evaluate the safety of DEX for clinical use in children with high quality, large sample, and multi-center retrospective study.

\section{Conclusion}

Our study indicates that incubation of hippocampal neurons with $100 \mu \mathrm{M}$ and $200 \mu \mathrm{M}$ DEX for $3 \mathrm{~h}$ reduced the activity of primary neurons, decreased the mitochondrial membrane potential, increased the expression of apoptosis-related proteins, and finally induced apoptosis in hippocampal neurons, which suggests that DEX has a dosedependent neurotoxic effect at high concentrations.

\section{Acknowledgments}

This work was supported by the Natural Science Foundation of Guangxi (No.AB18221031) and the National Natural Science Foundation of China (No. 81373498). We are grateful to the staff involved in this research, especially Ms. Zhou Sijia, for their concern, support, and understanding during our experiment and writing. 


\section{Author Contributions}

All authors made substantial contributions to conception and design, acquisition of data, or analysis and interpretation of data; took part in drafting the article or revising it critically for important intellectual content; agreed to submit to the current journal; gave final approval of the version to be published, and agree to be accountable for all aspects of the work.

\section{Disclosure}

The authors report no conflicts of interest in this work.

\section{References}

1. Hansen TG, Pedersen JK, Henneberg SW, et al. Academic performance in adolescence after inguinal hernia repair in infancy: a Nationwide Cohort Study. Anesthesiology. 2011;114:1076-1085. doi:10.1097/ALN.0b013e31820e77a0

2. Ko WR, Liaw Y, Huang J, et al. Exposure to general anesthesia in early life and the risk of attention deficit/hyperactivity disorder development: a Nationwide, Retrospective Matched-Cohort Study. Paediatr Anaesth. 2014;24:741-748. doi:10.1111/pan.12371

3. Shukla A, Chowdhary V. Neurodevelopmental outcome at 5 years of age after general anaesthesia or awake-regional anaesthesia in infancy (Gas): an international, multicentre, randomised, controlled equivalence trial. Acta Paediatr. 2019;108:2115-2116. doi:10.1111/ apa.14943

4. Sun LS, Li G, Miller TLK, et al. Association between a single general anesthesia exposure before age 36 months and neurocognitive outcomes in later childhood. JAMA. 2016;315(21):2312-2320. doi:10.1001/jama.2016.6967

5. Block RI, Thomas JJ, Bayman EO, Choi JY, Kimble KK, Todd MM. Are anesthesia and surgery during infancy associated with altered academic performance during childhood. Anesthesiology. 2012;117:494-503. doi:10.1097/ALN.0b013e3182644684

6. Flick RP, Katusic SK, Colligan RC, et al. Cognitive and behavioral outcomes after early exposure to anesthesia and surgery. Pediatrics. 2011;128(5):e1053-e1061. doi:10.1542/peds.2011-0351

7. Lng $\mathrm{CH}$, Dimaggio CJ, Malacova E, et al. Comparative analysis of outcome measures used in examining neurodevelopmental effects of early childhood anesthesia exposure. Surv Anesthesiol. 2015;59 (1):33-34. doi:10.1097/01.SA.0000459242.59331.b6

8. Wilder RT, Flick R, Sprung J, et al. Early exposure to anesthesia and learning disabilities in a population-based birth cohort Anesthesiology. 2009;110(4):796-804. doi:10.1097/01. anes.0000344728.34332.5d

9. Hayden JC, Breatnach C, Doherty DR, et al. Efficacy of A2-agonists for sedation in pediatric critical care: a systematic review. Pediatr Crit Care Med. 2016;17(2):e66-e75. doi:10.1097/ PCC. 0000000000000599

10. Mahmoud M, Mason KP. Dexmedetomidine: review, update, and future considerations of paediatric perioperative and periprocedural applications and limitations. Br J Anaesth. 2015;115(2):171-182. doi:10.1093/bja/aev226

11. Roback MG, Carlson DW, Babl FE, Kennedy RM. Update on pharmacological management of procedural sedation for children. Curr Opin Anaesthesiol. 2016;29(Supplement 1):S21-S35. doi:10.1097/ ACO.0000000000000316

12. Lee J-R, Lin EP, Hofacer RD, et al. Alternative technique or mitigating strategy for sevoflurane-induced neurodegeneration: a Randomized Controlled Dose-Escalation Study of Dexmedetomidine in neonatal rats. Br J Anaesth. 2017;119 (3):492-505. doi:10.1093/bja/aex219
13. Liu J-R, Yuki K, Baek C, Han X-H, Soriano SG. Dexmedetomidineinduced neuroapoptosis is dependent on its cumulative dose. Anesth Analg. 2016;123(4):1008-1017. doi:10.1213/ANE.00000000 00001527

14. Lyras L, Cairns NJ, Jenner AM, Jenner P, Halliwell B. An assessment of oxidative damage to proteins, lipids, and DNA in brain from patients with alzheimer's disease. $J$ Neurochem. 2002;68 (5):2061-2069. doi:10.1046/j.1471-4159.1997.68052061.x

15. Harada A, Teng J, Takei Y, Oguchi K, Hirokawa N. Map2 is required for dendrite elongation, pka anchoring in dendrites, and proper pka signal transduction. J Cell Biol. 2002;158(3):541-549. doi:10.1083/ jcb.200110134

16. Fredriksson A, Archer T, Alm H, Gordh T, Eriksson P. Neurofunctional deficits and potentiated apoptosis by neonatal nmda antagonist administration. Behav Brain Res. 2004;153 (2):367-376. doi:10.1016/j.bbr.2003.12.026

17. Satomoto M, Satoh Y, Terui K, et al. Neonatal exposure to sevoflurane induces abnormal social behaviors and deficits in fear conditioning in mice. Anesthesiology. 2009;110(3):628-637. doi:10.1097/ ALN.0b013e3181974fa2

18. Cattano D, Young C, Straiko MMW, Olney JW. Subanesthetic doses of propofol induce neuroapoptosis in the infant mouse brain. Anesth Analg. 2008;106(6):1712-1714. doi:10.1213/ane.0b013e318172ba0a

19. Istaphanous GK, Howard J, Nan X, et al. Comparison of the neuroapoptotic properties of equipotent anesthetic concentrations of desflurane, isoflurane, or sevoflurane in neonatal mice. Anesthesiology. 2011;114(3):578-587. doi:10.1097/ALN.0b013e3182084a70

20. Slikker W Jr, Zou X, Hotchkiss CE, et al. Ketamine-induced neuronal cell death in the perinatal rhesus monkey. Toxicol Sci. 2007;98 (1):145-158. doi:10.1093/toxsci/kfm084

21. Zou X, Liu F, Zhang X, et al. Inhalation anesthetic-induced neuronal damage in the developing rhesus monkey. Neurotoxicol Teratol. 2011;33(5):592-597. doi:10.1016/j.ntt.2011.06.003

22. Zou X, Patterson TA, Divine RL, et al. Prolonged Exposure to Ketamine Increases Neurodegeneration in the Developing Monkey Brain. Int $J$ Dev Neurosci. 2009;27(7):727-731. doi:10.1016/j. ijdevneu.2009.06.010

23. Pancaro C, Segal BS, Sikes RW, et al. Dexmedetomidine and ketamine show distinct patterns of cell degeneration and apoptosis in the developing rat neonatal brain. J Matern Fetal Neonatal Med. 2016;29 (23):3827-3833. doi:10.3109/14767058.2016.1148132

24. Shimizu S, Narita M, Tsujimoto Y, Tsujimoto Y. Bcl-2 family proteins regulate the release of apoptogenic cytochrome $\mathrm{C}$ by the mitochondrial channel Vdac. Nature. 1999;399(6735):483-487. doi: $10.1038 / 20959$

25. Orrenius S, Gogvadze V, Zhivotovsky B. Calcium and mitochondria in the regulation of cell death. Biochem Biophys Res Commun. 2015;460:72-81. doi:10.1016/j.bbrc.2015.01.137

26. Cui J, Zhao H. Dexmedetomidine attenuates oxidative stress induced lung alveolar epithelial cell apoptosis in vitro. Oxid Med Cell Longev. 2015;2015:358396.

27. Park JW, Chung HW, Lee EJ, Jung KH, Paik JY, Lee KH. A2-adrenergic agonists including xylazine and dexmedetomidine inhibit norepinephrine transporter function in Sk-N-Sh cells. Neurosci Lett. 2013;541:184-189. doi:10.1016/j.neulet.2013.02.022

28. Otera $\mathrm{H}$, Ishihara $\mathrm{N}$, Mihara $\mathrm{K}$. New insights into the function and regulation of mitochondrial fission. Biochim Biophys Acta. 2013;1833(5):1256-1268. doi:10.1016/j.bbamcr.2013.02.002

29. Carpio MA, Michaud M, Zhou W, Fisher JK, Walensky LD, Katz SG. Bcl-2 family member bok promotes apoptosis in response to endoplasmic reticulum stress. Proc Natl Acad Sci U S A. 2015;112 (23):7201-7206. doi:10.1073/pnas.1421063112

30. Zeng H, Kong X, Peng H, et al. Apoptosis and Bcl-2 family proteins, taken to chronic obstructive pulmonary disease. Eur Rev Med Pharmacol Sci. 2012;16(6):711-727. 
31. Gahl RF, Dwivedi P, Tjandra N. Bcl-2 proteins bid and bax form a network to permeabilize the mitochondria at the onset of apoptosis. Cell Death Dis. 2016;7(10):e2424. doi:10.1038/cddis.2016.320

32. Deng X, Gao F, May WS Jr. Bcl2 retards G1/S cell cycle transition by regulating intracellular ros. Blood. 2003;102(9):3179-3185. doi:10.1182/blood-2003-04-1027

33. Dispersyn G, Nuydens R, Connors R, Borgers M, Geerts H. Bcl-2 protects against Fccp-induced apoptosis and mitochondrial membrane potential depolarization in Pc12 cells. Biochim Biophys Acta. 1999;1428(2-3):357-371. doi:10.1016/S0304-4165(99)00073-2

34. Tang X-Q, Feng J-Q, Chen J, et al. Protection of oxidative preconditioning against apoptosis induced by $\mathrm{H} 2 \mathrm{o} 2$ in $\mathrm{Pc} 12$ cells: mechanisms via Mmp, Ros, and Bcl-2. Brain Res. 2005;1057(1-2):57-64. doi:10.1016/j.brainres.2005.07.072

35. Sena LA, Chandel NS. Physiological roles of mitochondrial reactive oxygen species. Mol Cell. 2012;48(2):158-167. doi:10.1016/j. molcel.2012.09.025

36. Turrens JF. Mitochondrial formation of reactive oxygen species. J Physiol. 2003;552(2):335-344. doi:10.1113/jphysiol.2003.049478

37. Brand MD, Affourtit C, Esteves TC, et al. Mitochondrial superoxide: production, biological effects, and activation of uncoupling proteins. Free Radic Biol Med. 2004;37(6):755-767. doi:10.1016/j. freeradbiomed.2004.05.034

38. Chen B-S, Peng H, Wu S-N. Dexmedetomidine, an $\alpha 2$-adrenergic agonist, inhibits neuronal delayed-rectifier potassium current and sodium current. Br J Anaesth. 2009;103(2):244-254. doi:10.1093/ bja/aep107
39. Ishii H, Kohno T, Yamakura T, Ikoma M, Baba H. Action of dexmedetomidine on the substantia gelatinosa neurons of the rat spinal cord. Eur $J$ Neurosci. 2008;27(12):3182-3190. doi:10.1111/j.14609568.2008.06260.x

40. Shirasaka T, Kannan H, Takasaki M. Activation of a G protein-coupled inwardly rectifying $\mathrm{K}+$ current and suppression of Ih contribute to dexmedetomidine-induced inhibition of rat hypothalamic paraventricular nucleus neurons. Anesthesiology. 2007;107:605-615. doi:10.1097/01.anes.0000281916.65365.4e

41. Creeley CE, Dikranian KT, Dissen GA, Back SA, Olney JW, Brambrink AM. Isoflurane-induced apoptosis of neurons and oligodendrocytes in the fetal rhesus macaque brain. Anesthesiology. 2014;120:626-638. doi:10.1097/ALN.0000000000000037

42. Andropoulos DB, Greene MF. Anesthesia and developing brains implications of the fda warning. $N$ Engl J Med. 2017;376:905-907. doi:10.1056/NEJMp1700196

43. Pia Banerjee MG, Rossi DL, Anghelescu WL, et al. Association between anesthesia exposure and neurocognitive and neuroimaging outcomes in long-term survivors of childhood acute lymphoblastic leukemia. JAMA Oncol. 2019;5:1456. doi:10.1001/jamaoncol. 2019.1094

44. Beaudoin GMJ, Lee S-H, Singh D, et al. Culturing pyramidal neurons from the early postnatal mouse hippocampus and cortex. Nat Protoc. 2012;7:1741-1754. doi:10.1038/nprot.2012.099

\section{Publish your work in this journal}

Drug Design, Development and Therapy is an international, peerreviewed open-access journal that spans the spectrum of drug design and development through to clinical applications. Clinical outcomes, patient safety, and programs for the development and effective, safe, and sustained use of medicines are a feature of the journal, which has also been accepted for indexing on PubMed Central. The manuscript management system is completely online and includes a very quick and fair peer-review system, which is all easy to use. Visit http://www. dovepress.com/testimonials.php to read real quotes from published authors. 